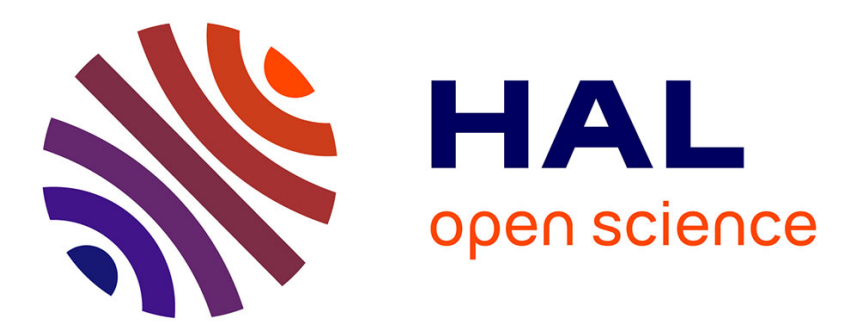

\title{
Modelling the vertical grating-waveguide coupler for the description of its nonlinear symmetry-breaking dynamics
}

M. Delqué, A. Dewandrea, K. Phan Huy, S.-P. Gorzaa, M. Haelterman

\section{To cite this version:}

M. Delqué, A. Dewandrea, K. Phan Huy, S.-P. Gorzaa, M. Haelterman. Modelling the vertical gratingwaveguide coupler for the description of its nonlinear symmetry-breaking dynamics. Optics Communications, 2009, 282 (17), pp.3607-3611. 10.1016/j.optcom.2009.05.076 . hal-00445470

\author{
HAL Id: hal-00445470 \\ https://hal.science/hal-00445470
}

Submitted on 13 Apr 2021

HAL is a multi-disciplinary open access archive for the deposit and dissemination of scientific research documents, whether they are published or not. The documents may come from teaching and research institutions in France or abroad, or from public or private research centers.
L'archive ouverte pluridisciplinaire HAL, est destinée au dépôt et à la diffusion de documents scientifiques de niveau recherche, publiés ou non, émanant des établissements d'enseignement et de recherche français ou étrangers, des laboratoires publics ou privés.

\section{(c)(1)}

Distributed under a Creative Commons Attribution| 4.0 International License 


\title{
Modelling the vertical grating-waveguide coupler for the description of its nonlinear symmetry-breaking dynamics
}

\author{
M. Delqué ${ }^{\mathrm{a}, *}$, A. Dewandre ${ }^{\mathrm{a}}$, K. Phan Huy ${ }^{\mathrm{b}}$, S.-P. Gorza ${ }^{\mathrm{a}}$, M. Haelterman ${ }^{\mathrm{a}}$ \\ a Service OPERA-Photonique, CP194/5, Université Libre de Bruxelles (ULB), Avenue F.D. Roosevelt, 1050 Bruxelles, Belgium \\ ${ }^{\mathrm{b}}$ Département d'Optique P.M. Duffieux, Institut FEMTO-ST, Université de Franche-Comté, CNRS UMR 6174, 25030 Besançon, France
}

\begin{abstract}
We propose a new scheme to observe the symmetry-breaking dynamics of two counter-propagating beams in a Kerr-type nonlinear waveguide coupler. Using a grating to couple light at normal incidence upon the waveguide allows for a single-beam geometry. The ability of the grating to couple light symmetrically is demonstrated on the basis of a linear coupled-mode approach especially adapted to vertical coupling. The nonlinear symmetry-breaking dynamics is studied analytically.
\end{abstract}

\section{Introduction}

Kerr-type nonlinear resonators have been widely studied in the past for the richness of their dynamics. These devices were indeed shown to exhibit a rich spectrum of complex behaviors such as bistability, multistability, self-pulsing, modulational instabilities and chaos [1-3]. In addition to these phenomena, symmetry-breaking instabilities have been predicted theoretically [4] as soon as different field components enter into competition. In most systems two beams are used with different but symmetric directions with respect to the resonator axis [5]. In certain configurations the two fields are merely the two polarization components of the same laser beam [6]. In these twin-field systems, the nonlinear coupling of the two fields inside the resonator induces a competition between them that in turn leads to a symmetry-breaking instability. This process is of particular potential interest as regards applications because it can be exploited as the basic physical mechanism of alloptical flip-flop devices useful in ultrafast optical signal processing.

The principle of twin-field nonlinear resonators has been proposed under a variety of configurations involving for instance, fiber loop cavities [6] or coupled micro-cavities [7]. One of the first configurations proposed in the literature is the bulk Fabry-Pérot cavity where two beams are used to symmetrically pump two counter-

\footnotetext{
* Corresponding author. Tel.: +32 2650 3203; fax: +32 26504496

E-mail address: Michael.Delque@ulb.ac.be (M. Delqué).
}

propagating resonator modes [5]. However, despite its conceptual simplicity, the physical implementation of this system is truly challenging because of the required accuracy in the symmetry of the twin-pumping process and, to our knowledge, no experiments were performed to demonstrate flip-flop operation.

In response to this difficulty, we propose in the present paper a simpler and much more realistic scheme that in principle should lead to easy experimental demonstrations of optical symmetrybreaking dynamics. In our system a grating is used to symmetrically couple a single light beam in a nonlinear waveguide. The system made of the waveguide and the grating constitutes the twin-field nonlinear resonator (see Fig. 1a). Gratings have already been proposed in bistable switches as nonlinear reflectors [8] or as waveguide couplers through which beams at oblique incidence are injected [9]. The essential difference with our system is that we consider a grating adapted to the coupling of an external beam coming at normal incidence on the planar waveguide surface. Normal incidence, which requires that the grating period matches the wavelength of the waveguide modes, leads to symmetric coupling of the counter-propagating modes. This system is thus naturally adapted to the study of symmetry-breaking dynamics. Note that vertical coupling schemes are well-known and already used for many applications [10-13], in particular in the study of the bistable nonlinear response of photonic crystals resonators [14].

In Section 2, we propose a theoretical description of the normal incidence grating coupler in the linear regime. A coupled-mode 


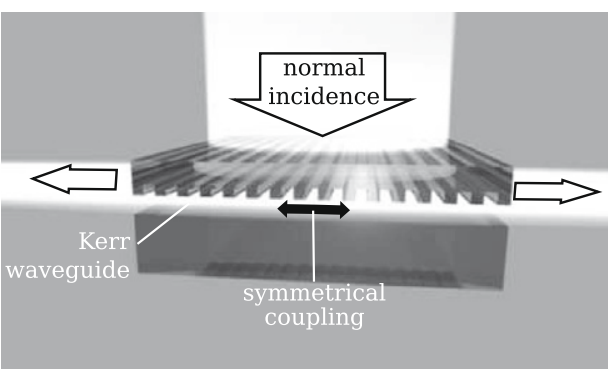

(a)

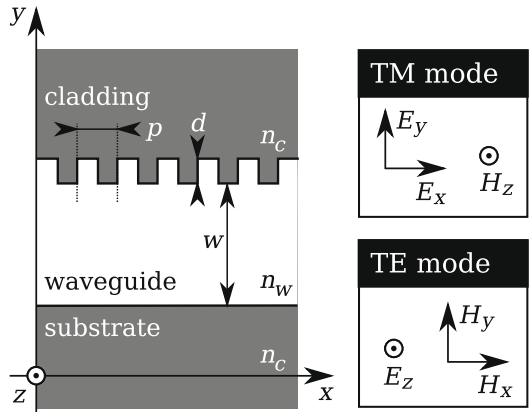

(b)

Fig. 1. Single-beam vertical grating-waveguide coupler allowing symmetrical pumping and nonlinear symmetry-breaking: principle (a) and detailed configuration (b).

approach is used and derived for the first time to our knowledge to describe vertical coupling to both counter-propagating modes. This theory, which is based on a semi-analytical model, allows us to fully characterize the linear resonance of the device. In Section 3, nonlinearity is introduced in a phenomenological way, which leads us to a model similar to that of the twin-beam nonlinear FabryPérot resonator of Ref. [5]. The symmetry-breaking dynamics of the device is then demonstrated.

\section{Linear modeling}

\subsection{Numerical model}

The principle and the structure of the normal incidence grating coupler device are sketched in Fig. 1. The structure consists of a $w=1 \mu \mathrm{m}$-thick planar waveguide sandwiched between a substrate and a grating engraved cladding of refractive index $n_{c}$ (gray area in Fig. 1b). The guiding layer is an ideal Kerr medium (white area) whose linear refractive index is $n_{w}>n_{c}$. The period and depth of the grating are $p$ and $d$, respectively, and its duty cycle is 0.5 . The vertical cavity is oriented along the $y$ direction while the field can freely propagate along the $x$ axis.

Due to the normal incidence, standard coupled-mode techniques (CMT) cannot be exploited to describe the coupling of light in our structure. The reason is that in the CMT, one should solve Maxwell's equations for the optical waveguide (guided modes) and free space (plane waves). This theory thus describes the grating-induced coupling between the guided modes and the scattered plane waves. The corresponding coupling coefficient is then proportional to the overlap $\int_{S} \psi_{1} \psi_{2}^{\star} d s$, where $S$ is the plane orthogonal to the waveguide axis and $\psi_{1}, \psi_{2}$ stand for the wave functions of, respectively, the guided mode and the plane wave [15]. However, this overlap makes no sense as long as the plane wave propagates at normal incidence with respect to the waveguide axis. This means that the first models based on CMT were dedicated to the coupling with oblique incidence. Later, these methods have been improved in the framework of distributed feedback (DFB) and distributed Bragg reflector (DBR) lasers $[16,17]$ by taking the second order diffraction terms into account. In these works, coupling to $90^{\circ}$ out-of-plane radiation were considered but computation of the associated coupling coefficient remains complicated.

To obtain these coupling coefficients, we have therefore developed a new theoretical approach adapted to the normal incidence coupling. Compared to standard models based on CMT, our approach is rather straightforward. We solve Maxwell's equations for the whole cavity including the optical waveguide, the grating and its surroundings, which enables us to describe the resonant modes of this cavity. We then focus on one specific resonant mode that scatters the light vertically from the resonator. Thanks to this method, one can readily obtain the electric and magnetic fields, the resonant wavelengths and the quality factors for both polarizations.

Given the multi-layer geometry, the transverse electric and magnetic fields (TE and TM, respectively) are defined by their non-zero components represented on the right side of Fig. $1 \mathrm{~b}$. To characterize the linear behavior of the structure, we use a Floquet-Bloch expansion of the electric and magnetic fields on a single grating period [18]:

$X_{i}(x, y, t)=\sum_{h} X_{i}^{h} e^{j h \beta_{G} x} e^{j \sigma_{h y} y} e^{j \omega t}+$ c.c.

where $h=0,1,2,3, \ldots ; \beta_{G}=2 \pi / p ; \sigma_{h, y}^{2}=(\omega n(y) / c)^{2}-\left(h \beta_{G}\right)^{2}$ and $\beta_{h}$ is the wave number of the $h$ th harmonic. $X_{i}$ stands for the electric or the magnetic field components $\left(X_{i}=E_{i}, H_{i}\right.$ and $\left.i=x, y, z\right)$ and c.c. denotes the complex conjugate. The weight coefficients $X_{i}^{h}$ and the refractive index $n_{y}$ are different in each zone labeled as in Fig. 1b (cladding, waveguide, grating and substrate). To ensure the computation of a guided mode, the sign of $\sigma_{h y}$ in the cladding and the substrate is defined by appropriate boundary conditions.

Developing Maxwell's equations results in a coupling between the harmonics of the fields in the different layers of the structure. Every $h$ th field harmonic is coupled with the others through matrices representing propagation in the different layers and through continuity conditions at the interfaces $[18,19]$. This procedure defines an eigenvalue problem which can be numerically solved to obtain the $X_{i}$ values and the eigenvalue $\lambda=2 \pi \mathrm{c} / \omega$. Starting from a real value as initial guess for $\lambda$, the solution converges to a complex value. The real part of $\lambda$ gives the wavelength of the resonance mode. The imaginary part of $\lambda$, which accounts for the waveguide losses due to the grating structure [18], sets the quality factor $Q=|\lambda| /[2 \operatorname{Im}(\lambda)]$ of the resonant structure for a given mode.

A typical result for a monomode waveguide at a wavelength $\mathfrak{R e}(\lambda)=1.5 \mu \mathrm{m}$ is shown in Fig. 2 where the profile of the transverse magnetic (TM) and electric (TE) resonance modes are represented. Monomode property ensures that nonlinear coupling effect only exists between counter-propagating modes. To achieve resonant coupling, the period of the grating has to match the index of the structure's mode, leading to a grating period of $p=\lambda / n_{\text {mode }}$. Such a period is reminiscent of a guided wave coupled to $90^{\circ}$ radiation through the first order diffraction. One can see here the fundamental difference between this kind of coupler and Bragg reflectors (BR), in which counter-propagating waves are coupled through the first order diffraction, i.e. for grating period $p_{B R}=\lambda /\left(2 n_{\text {mode }}\right)$.

This figure clearly shows two fundamental features of the TM mode:

(1) Inside the waveguiding layer, $X_{w}^{h} / X_{w}^{ \pm 1}<0.007$ for $h \neq \pm 1$ in the TM mode expansion. This means that the shape of this mode is mainly determined by the $X_{w}^{ \pm 1}$ components. 


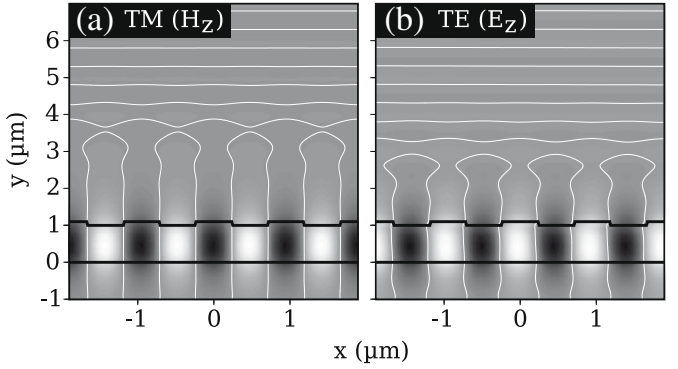

Fig. 2. Numerically calculated transverse magnetic (a) and electric (b) modes of the structure. The real part of the fields is represented in grayscale and the nodes are enhanced in white line to distinguish the wavefronts. The bold black lines depict the structure. Parameters are $\mathfrak{R e}(\lambda)=1.5 \mu \mathrm{m} \quad$ (optical wavelength), $n_{c}=1.5, n_{w}=1.64, p=0.95 \mu \mathrm{m}, d=0.1 \mu \mathrm{m}$. The duty cycle of the grating is 0.5 . These computations were performed with seven harmonics in the Floquet-Bloch expansion.

(2) The energy trapped inside the waveguide can escape through a plane wave which is the $h=0$ component of the field's expansion inside the cladding $\left(X_{c}^{0}\right.$ in Eq. (1)). One can see that this pumping plane wave propagates at $90^{\circ}$ with respect to the waveguide axis. Higher harmonics (i.e. $h \neq 0$ ) in the claddings are actually evanescent since they are characterized by an imaginary value of $\sigma_{y}$. This confirms that light naturally escapes from the structure at normal incidence or, inversely, that external light at normal incidence can feed the fundamental TM mode.

Note that these results will be used to derive a good approximation of the field in our analytical model described in Section 2.2. Indeed, the expansion of the guided and pumping fields will be restricted to $X_{w}^{ \pm 1}$ and $X_{\text {input }}^{0}=X_{c}^{0}$, respectively.

Because of the strong spatial asymmetry induced by the grating, the TE and TM mode profiles are not identical, which is reflected by their different modal indices as well as the different wavevectors of their plane wave components within the cladding and the substrate. This is illustrated in Fig. $2 b$ that reveals an angle of incidence of $90-0.5^{\circ}$ for the plane wave component of the TE mode. Note that this angle appears when only the right propagating harmonics are taken into account for drawing the figure. Left propagating modes give the symmetric situation with a $90+0.5^{\circ}$ angle of incidence. Carefully chosen coupling conditions thus enable us in principle to achieve optimal injection for the TM mode alone, since the TE mode is then off-resonance. In addition to this difference in coupling conditions, there is a strong discrimination between the TE and TM modes because of their unequal quality factors $\left(Q_{\mathrm{TE}}=\right.$ $2.0 \times 10^{2}$ and $Q_{\mathrm{TM}}=2.7 \times 10^{5}$ ). This three order of magnitude difference means that the energy will preferentially be accumulated in TM mode and that any energy transferred to the TE mode through nonlinear coupling will merely result in additional losses, since this energy is rapidly radiated outside the cavity. As a result, one can reasonably expect that the TM polarization is conserved in the nonlinear regime, which prevents the polarization instability mechanism described in Ref. [6]. Accordingly, in the following we will only consider the TM mode of the guiding structure.

In summary, we can state that the grating allows for the symmetrical coupling of the two counter-propagating TM guided modes in the absence of any change of polarization state and that, consequently, the conditions to observe symmetry breaking between these two guided modes are fulfilled.

\subsection{Analytical description}

Obviously, the modal approach presented above cannot account for the waveguide nonlinearity. The aim of the present section is to derive a simple model that allows for the inclusion of the mode parameters computed previously and of nonlinearity in a phenomenological way.

The numerical model derived in the previous section provides a discrete set of leaky modes characterized by their coupling or leaking coefficient $\alpha=\mathfrak{I}\left(2 \pi n_{\text {mode }} / \lambda\right)$. They represent the different diffraction orders of the grating. As shown in Refs. [20,21], thanks to their orthogonality properties and once properly normalized, these modes can be used to describe the radiation spectrum of waveguiding structures, though they do not constitute a basis for any electromagnetic solution. The leaky modes write

$\mathscr{E}_{k}(x, y, t)=E_{k}(x, y) e^{\left(j \omega_{k}-\alpha_{k}\right) t}+$ C.C.,

where $\omega_{k}$ and $\alpha_{k}$ are the pulsation and the loss factor related to the resonance width of the $k$ th mode, respectively. These modes are solution of the Maxwell's equations solved numerically in the previous section. In the monomode structure defined in the previous section, $k$ is reduced to 1 , this is the reason why the $k$ numbering is removed.

We restrict ourselves to the study of the amplitude of the electromagnetic field inside the guiding layer and not of the whole mode which extends also outside this layer. Such quasi-monochromatic coupling model of slowly varying amplitude [16,22,25] writes

$\frac{\partial E^{\prime}(x, y)}{\partial x}+j \beta E^{\prime}(x, y)-\alpha E^{\prime}(x, y)=-\alpha f(P(x, y))$,

where $E^{\prime}(x, y)$ stands for the field inside the guiding layer and the pumping term is expressed as a function $f$ of the pump beam $P(x, y) . P(x, y)$ represents the superstrate part of the leaky mode and $f$ defines how efficiently it is coupled to $E^{\prime}(x, y)$. Indeed we know, thanks to the mode computation detailed in the previous section, how to totally describe a leaky mode starting from the superstrate components only.

As we have seen in Section 2.1, the fundamental components $h= \pm 1$ are the dominant terms of the Floquet-Bloch expansion of $E^{\prime}(x, y)$ inside the waveguide. We can therefore reasonably neglect the high order harmonics $(h \neq \pm 1)$, which gives

$E^{\prime}(x, y)=E^{+}(x, y)+E^{-}(x, y)$,

where $E^{ \pm}(x, y)$ varies in the $x$ direction only through $e^{ \pm j \beta_{G} x}$ [see Eq. (1)].

Since the $e^{ \pm j \beta_{G} x}$ functions are mutually orthogonal, in the following we will only consider the evolution of the forward propagating component $E^{+}(x, y)=X_{w}^{+1} e^{+j \beta_{G} x} e^{j \sigma_{y} y}$. Distributed feedback effect [23] in the waveguide direction could result in linear coupling between counter-propagating modes $E^{+}(x, y)$ and $E^{-}(x, y)$. This coupling is possible through the second order diffraction of the grating. However, as the grating has a rectangular shape with the low and the high index parts having the same length ( 0.5 duty cycle), this coupling does not occur [22]. The corresponding coupling coefficient is indeed proportional to the second Fourier coefficient of the index modulation [16], which is equal to 0 for such a duty cycle. This is the reason why this effect is not included in the present work. Eq. (3) becomes

$\frac{\partial E^{+}(x, y)}{\partial x}+j \beta_{G} E^{+}(x, y)-\alpha E^{+}(x, y)=-\alpha f^{+}(P(x, y))$

$f^{+}$is the coupling function $f$ restricted to $E^{+}(x, y)$ coupling.

Using once again the results of the mode computation described in Section 2.1, we know that the phase-matched pumping field can be approximated to the normally incident input plane wave. In the right-hand side of Eq. (5), $f^{+}(P(x, y))$ is then replaced by

$f^{+}\left(X_{\text {input }}^{0}\right)$ 
with $f^{+}$defined by the matrix system used in the modal approach of the structure (see Section 2.1). $X_{\text {input }}^{0}$ is the weight of the zero order harmonic (plane wave at normal incidence) of the field in the superstrate. This is in accordance with the results derived in the previous section which show the plane wave character of the far field in the superstrate. It therefore clearly appears that the $f^{+}$function defines the coupling efficiency between the vertical input plane wave and the modes of the waveguide. Given our approximation, i.e. when only the first order harmonic $(h=+1)$ inside the waveguide is taken into account, $f^{+}\left(X_{\text {input }}^{0}\right) \simeq X_{w}^{+1} e^{+j \beta_{G} x} e^{j \sigma_{y} y} \simeq K X_{\text {input }}^{0} e^{+j \beta_{G} x} e^{j \sigma_{y} y}$, where $X_{w}^{+1}$ is the amplitude defined in Eq. (1) and $K$ is a constant. In the same configuration as in Fig. 2, numerical simulations provide the value $K \simeq 200$. This large value is the result of the resonant nature of the coupling and is naturally associated with the large quality factor. Note that the same reasoning applies to the backward field $E^{-}$.

Eq. (5) becomes:

$\frac{\partial E^{+}(x, y)}{\partial x}+j \beta_{G} E^{+}(x, y)-\alpha E^{+}(x, y)=-\alpha K X_{\text {input }}^{0} e^{+j \beta_{G} x} e^{j \sigma_{y} y}$.

Let us now consider a small detuning $\delta \beta=\beta_{G}-\beta \ll \beta$ induced by a small change in the wavelength of the incident light so as to preserve the symmetry of the coupling process (namely, normal incidence coupling). We will assume that the incident field has an angular spectrum sufficiently narrow to excite only the $k= \pm 1$ modes. The perturbed field in the system can therefore be written as $R(x) E^{+}(x, y)$, which satisfies the field continuity conditions. Eq. (7) thus becomes:

$$
\begin{aligned}
& \frac{\partial R(x)}{\partial x} E^{+}(x, y)+j\left(\beta-\beta_{G}\right) R(x) E^{+}(x, y)-\alpha R(x) E^{+}(x, y) \\
& \quad=-\alpha K X_{\text {input }}^{0} e^{+j \beta_{G} x} e^{j \sigma_{y} y}
\end{aligned}
$$

Taking into account the complex conjugate terms, we can replace $E^{+}(x, y)$ by $U \cos \left(\sigma_{y} y+\phi\right) e^{+j \beta_{G} x}$. Eq. (8) thus becomes

$\frac{\partial R(x)}{\partial x}+j\left(\beta-\beta_{G}\right) R(x)-\alpha R(x)=-\alpha K^{\prime} X_{\text {input }}^{0}$

with dimensionless factor $K^{\prime}=K / U$. In the following, we will set the amplitude $U$ equal to $1 \mathrm{~V} / \mathrm{m}$, the amplitude of the electric field being included in the dimensionless envelope $R(x)$.

\section{Nonlinear dynamics}

To study the nonlinear behavior of the waveguide grating coupler in a way close to previous nonlinear coupling schemes [24-26], a perturbation theory is applied to the linear model developed in the previous section. In this approach we take the Kerr nonlinearity into account by replacing $\beta$ by $\beta+\gamma|R(x)|^{2}$, where $\gamma=(2 \pi / \lambda) n_{2} / n_{w}, n_{2}$ being the nonlinear index coefficient of the waveguiding layer. Following the perturbation method described in Refs. [27,28], the perturbative term $\gamma|R(x)|^{2}$ leads to new eigenvalues $\beta_{\mathrm{NL}}$ of the nonlinear problem:

$\beta_{\mathrm{NL}}=\beta_{G}-\frac{3}{8} \gamma|R(x)|^{2}$.

Eq. (9) thus becomes

$\frac{\partial R(x)}{\partial x}+j\left(\beta-\beta_{G}+\frac{3}{8} \gamma|R(x)|^{2}\right) R(x)-\alpha R(x)=-\alpha K^{\prime} X_{\text {input }}^{0}$.

When the two counter-propagating modes $R(x)$ and $L(x)$, both pumped by the normally incident plane wave, are considered, nonlinear effect does not only consist in self-phase modulation $\gamma|R(x)|^{2}$ but also in cross-phase modulation [29] proportional to $\gamma|L(x)|^{2}$. This latter term couples the counter-propagating fields through nonlinear Kerr effect:

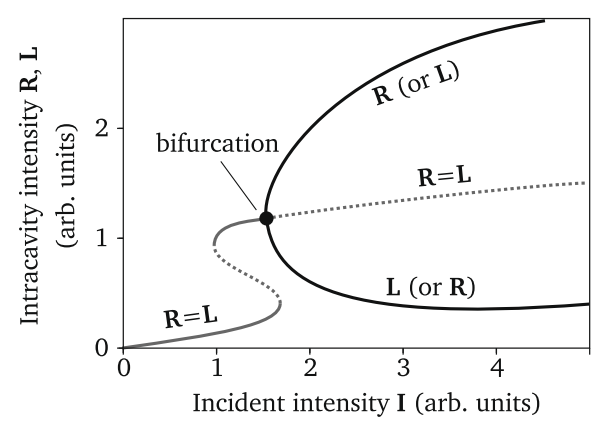

Fig. 3. Bifurcation diagram: output intensities $(\mathbf{R}, \mathbf{L})$ versus input intensity I Continuous line stands for stable states and dotted line for unstable ones. $\Delta=3$.

$$
\frac{\partial R(x)}{\partial x}+j\left[\beta-\beta_{G}+\frac{3}{8} \gamma\left(|R(x)|^{2}+2|L(x)|^{2}\right)\right] R(x)-\alpha R(x)=-\alpha K^{\prime} X_{\text {input }}^{0},
$$$$
\frac{\partial L(x)}{\partial x}+j\left[\beta-\beta_{G}+\frac{3}{8} \gamma\left(|L(x)|^{2}+2|R(x)|^{2}\right)\right] L(x)-\alpha L(x)=-\alpha K^{\prime} X_{\text {input }}^{0} .
$$

In this system, the ratio between cross-phase modulation and selfphase modulation equals to 2 . This value accounts for the coupling between the two counter-propagating modes through the local Kerr nonlinearity of the waveguide [29].

As expected, the system of Eq. (12) is equivalent to the analytical model of the nonlinear Fabry-Pérot derived in Ref. [5]. We can therefore reasonably expect that our system will also present a symmetry-breaking pitchfork bifurcation in the nonlinear regime of operation. Indeed, in the frame of the infinite plane wave approximation, following Ref. [5], we can easily derive from Eq. (12) the following set of coupled algebraic equations:

$(\mathbf{R}-\mathbf{L})\left[\mathbf{R}^{2}+\mathbf{R L}+\mathbf{L}^{2}-2 \Delta(\mathbf{L}+\mathbf{R})+\Delta^{2}+1\right]=0$,

where $(\mathbf{R}, \mathbf{L})=3 / 8(\gamma / \alpha)\left(|R|^{2},|L|^{2}\right)$ and $\Delta=\left(\beta-\beta_{G}\right) / \alpha$. We recall that Eq. (14) is obtained by subtracting the couple of equations which is derived by taking the square modulus of Eq. (13).

The resolution of Eq. (14) gives the bifurcation diagram depicted in Fig. 3 which represents $(\mathbf{R}, \mathbf{L})$ steady states as a function of the input intensity $\mathbf{I}=3 / 8(\gamma / \alpha) K^{\prime 2}\left|X_{\text {input }}^{0}\right|^{2}$. At low input power we see a S-shape curve that corresponds to a bistable but symmetrical regime $(\mathbf{R}=\mathbf{L})$. For higher input power, the symmetric solution becomes unstable and the system bifurcates into the asymmetric solutions $(\mathbf{R}<>\mathbf{L})$, which reveals a symmetry-breaking instability. As it was demonstrated in Ref. [5], observation of symmetry breaking requires $\Delta>\sqrt{3}$.

In certain conditions the right beam $\mathbf{R}$ could adopt, for instance, the higher branch at the pitchfork bifurcation threshold. If the input intensity is then increased, the contrast between the intensities of the counter-propagating modes increases, which means that the right beam is predominant in the waveguide. In this way, the waveguide emits more light from its right output end than from its left output end. The device could be switched to the opposite stable state $(\mathbf{R}<\mathbf{L})$ thanks to non symmetrical stimuli [6,7] such as an oblique incidence pulse injection. The configuration considered above and depicted in Fig. 2 can be made by encapsulating a Kerr liquid between an engraved grating and a flat substrate made of glass. With carbon disulfide as the nonlinear medium $\left(n_{2}=3.5 \times\right.$ $10^{-18} \mathrm{~W} \mathrm{~m}^{-2}$ ) the bifurcation point in Fig. 3 corresponds approximatively to a continuous wave incident intensity of $4 \mathrm{~W} \mathrm{~cm}^{-2}$.

\section{Conclusion}

In this paper we proposed a simple scheme to observe the leftright symmetry breaking between counter-propagating modes in 
Kerr-type waveguides. We demonstrated that a grating coupler designed for use at normal incidence leads, in the linear regime, to symmetrical excitation of the two counter-propagating modes; a condition that is necessary to obtain symmetry-breaking dynamics in the nonlinear regime. Thanks to a simplified model of the device based on Floquet-Bloch mode expansion and a phenomenological inclusion of the Kerr nonlinearity, we have indeed demonstrated the existence of a symmetry-breaking instability at relatively low input power.

In conclusion we have identified a structure that could be exploited to realize realistic ultrafast all-optical flip-flop devices. Experimental demonstrations based on liquid nonlinear layer (carbon disulfide) is in progress.

\section{Acknowledgements}

This work is supported by the Interuniversity Attraction Pole Program of the Belgian Government under Grant IAP6-10 and by a ULB fellowship. This work benefits from the Programme International de Coopération Scientifique PICS-3742 of the French Centre National de la Recherche Scientifique (CNRS). A part of this work was supported by the Groupement de Recherche Photonique Nonlinéaire et Milieux Microstructurés GDR-3073 created by CNRS. Kien Phan Huy thanks the Conseil Régional de Franche-Comté.

\section{References}

[1] A. Szöke, V. Daneu, J. Goldhar, N.A. Kurnit, Appl. Phys. Lett. 15 (11) (1969) 376

[2] H.M. Gibbs, S.L. McCall, T.N.C. Venkatesan, Phys. Rev. Lett. 36 (19) (1976) 1135.

[3] K. Otsuka, K. Ikeda, Opt. Lett. 12 (8) (1987) 599.
[4] T. Yabuzaki, T. Okamoto, M. Kitano, T. Ogawa, Phys. Rev. A 29 (4) (1984) 1964.

[5] M. Haelterman, P. Mandel, Opt. Lett. 15 (23) (1990) 1412.

[6] J. García-Mateos, F. Canal, M. Haelterman, Opt. Commun. 137 (1997) 427.

[7] B. Maes, M. Soljacic, J.D. Joannopoulos, P. Bienstman, R. Baets, S.-P. Gorza, M. Haelterman, Opt. Exp. 14 (22) (2006) 10678.

[8] U. Peschel, T. Peschel, F. Lederer, D. Berard, P. Dansas, N. Paraire, J. Opt. Soc. Am. B 12 (7) (1995) 1249.

[9] I. Avrutsky, A.M. Prokhorov, V.A. Sychugov, Sov. Lightwave Commun. 1 (1991) 71.

[10] A.A. Erchak, D.J. Ripin, S. Fan, P. Rakich, J.D. Joannopoulos, E.P. Ippen, G.S. Petrich, L.A. Kolodziejski, Appl. Phys. Lett. 78 (5) (2001) 563.

[11] D. Taillaert, W. Bogaerts, P. Bienstman, T. Krauss, P. Van Daele, I. Moerman, S. Verstuyft, K. De Mesel, R. Baets, IEEE J. Quant. Electron. 38 (7) (2002) 949.

[12] B. Wang, J. Jiang, G.P. Nordin, IEEE Photon. Technol. Lett. 17 (9) (2005) 1884.

[13] G. Roelkens, D.V. Thourhout, R. Baets, Opt. Lett. 32 (11) (2007) 1495.

[14] A.M. Yacomotti, F. Raineri, G. Vecchi, P. Monnier, R. Raj, A. Levenson, B.B. Bakir, C. Seassal, X. Letartre, P. Viktorovitch, L.D. Cioccio, J.M. Fedeli, Appl. Phys. Lett. 88 (23) (2006) 231107.

[15] A. Yariv, IEEE J. Quant. Electron. 9 (9) (1973) 919.

[16] W. Streifer, D. Scifres, R. Burnham, IEEE J. Quant. Electron. 13 (4) (1977) 134.

[17] A. Hardy, D. Welch, W. Streifer, IEEE J. Quant. Electron. 25 (10) (1989) 2096

[18] K.C. Chang, V. Shah, T. Tamir, J. Opt. Soc. Am. 70 (7) (1980) 804.

[19] K. Phan Huy, A. Morand, P. Benech, IEEE J. Quant. Electron. 41 (3) (2005) 357.

[20] K. Phan Huy, A. Morand, D. Amans, P. Benech, J. Opt. Soc. Am. B 22 (8) (2005) 1793.

[21] P. Bienstman, R. Baets, Opt. Quant. Electron. 34 (5) (2002) 523.

[22] R. Kazarinov, C. Henry, IEEE J. Quant. Electron. 21 (2) (1985) 144.

[23] H. Kogelnik, C.V. Shank, Appl. Phys. Lett. 18 (4) (1971) 152

[24] G.M. Carter, Y.J. Chen, Appl. Phys. Lett. 42 (8) (1983) 643.

[25] M. Haelterman, G. Vitrant, R. Reinisch, J. Opt. Soc. Am. B 7 (7) (1990) 1309.

[26] G. Vitrant, R. Reinisch, J.C. Paumier, G. Assanto, G.I. Stegeman, Opt. Lett. 14 (16) (1989) 898.

[27] C. Cohen-Tannoudji, B. Diu, F. Laloë, Mécanique Quantique, Hermann, Paris, 1998.

[28] G.P. Agrawal, Nonlinear Fiber Optics, third ed., Optics and Photonics, Academic Press, San Diego, 1995.

[29] S. Pitois, G. Millot, S. Wabnitz, J. Opt. Soc. Am. B 18 (4) (2001) 432. 